\title{
Determination of effective mass of heavy hole from phonon-assisted excitonic luminescence spectra in $\mathrm{ZnO}$
}

\author{
S. L. Shi ${ }^{\mathrm{a})}$ and S. J. Xu ${ }^{\mathrm{b})}$ \\ Department of Physics and HKU-CAS Joint Laboratory on New Materials, The University of Hong Kong, \\ Pokfulam Road, Hong Kong, China
}

(Received 29 September 2010; accepted 16 December 2010; published online 10 March 2011)

\begin{abstract}
Longitudinal optical (LO) phonon-assisted luminescence spectra of free excitons in high-quality $\mathrm{ZnO}$ crystal were investigated both experimentally and theoretically. By using the rigorous Segall-Mahan model based on the Green's function, good agreement between the experimental emission spectra involving one or two LO phonons and theoretical spectra can be achieved when only one adjustable parameter (effective mass of heavy hole) was adopted. This leads to determination of the heavy-hole effective mass $m_{h \perp}=\left(0.8 m_{0}\right.$ and $\left.m_{h \|}=5.0 m_{0}\right)$ in $\mathrm{ZnO}$. Influence of anisotropic effective masses of heavy holes on the phonon sidebands is also discussed. (C) 2011 American Institute of Physics. [doi:10.1063/1.3549724]
\end{abstract}

\section{INTRODUCTION}

Zinc oxide $(\mathrm{ZnO})$ has received a renewable great deal of interest in recent years due to its unique physical properties and potential technological applications in the new kind of exciton-based photonic devices. ${ }^{1-5}$ An essential reason for developing $\mathrm{ZnO}$-based photonic devices is that the extremely large binding energy $(\sim 60 \mathrm{meV})$ of free excitons in $\mathrm{ZnO}^{5-7}$ can lead to dominant excitonic radiative recombination at room temperature or even higher temperatures. Thus fabrication of $\mathrm{ZnO}$-based photonic devices with higher quantum efficiency and lower threshold pumping energy is a straightforward expectation. However, such expectation may be challenged by the strong coupling of free excitons with longitudinal optical (LO) phonons in $\mathrm{ZnO}^{8-11}$ For example, Shan et al. experimentally show that the LO-phonon-assisted emission rather than direct radiative recombination of the free excitons actually dominates at the room-temperature photoluminescence (PL) spectrum of $\mathrm{ZnO} .^{12}$ Full exploitation of $\mathrm{ZnO}$-based photonic devices certainly demands a better understanding of fundamental optical properties such as exciton-phonon interactions and their role in the light emission of free excitons in $\mathrm{ZnO}$. Therefore, a detailed investigation of temperature-dependent LO phonon sidebands (PSBs) of free excitons in $\mathrm{ZnO}$ is indispensable. In this paper, we report a combined theoretical and experimental study of the temperature-dependent LO-PSBs of free excitons in $\mathrm{ZnO}$. In particular, anisotropic effective masses of heavy hole in $\mathrm{ZnO}$ are determined. ${ }^{13,14}$

\section{EXPERIMENTAL AND SEGALL-MAHAN THEORETICAL MODEL}

The $\mathrm{ZnO}$ samples used in the present study were commercial bulk crystals (Commercial Crystal Laborato-

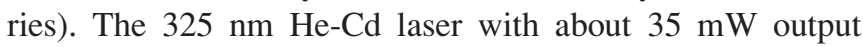

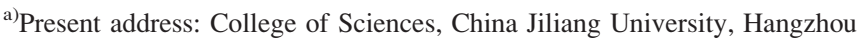
310018, China.

b) Author to whom correspondence should be addressed. Electronic mail: sjxu@hkucc.hku.hk.
}

power was employed to illuminate the $\mathrm{Zn}$-terminated (0001) surface of the sample at an incident angle of about $45^{\circ}$. During the variable-temperature PL measurements, the sample was mounted on the cold finger of a Janis closed cycle cryostat with the varying temperature range of 4-300 K. The emission signal was dispersed by a SPEX 750M monochromator with 1200 lines/mm grating and detected by a Hamamatsu R928 detector. Standard lock-in amplifier technique was also employed to enhance the signal-to-noise ratio.

In order to achieve a deeper understanding of the temperature-dependent LO PSBs of free excitons in $\mathrm{ZnO}$, we first calculate the first-order (1LO) and the second-order (2LO) PSBs of free excitons in $\mathrm{ZnO}$ using the Segall-Mahan (S-M) theory. ${ }^{15,16}$ The validity and effectiveness of the S-M model in the calculations of the LO phonon-assisted transitions of free excitons in wide gap polar semiconductors have been recently checked in GaN which is another wide gap semiconductor with wurtzite structures. ${ }^{17,18}$ According to the S-M theory, the absorption coefficients for the processes involving one phonon and two phonons can be calculated by ${ }^{15,16}$

$$
\begin{gathered}
\alpha_{1}(\omega)=\frac{4 \pi e^{2} \beta_{A, 1} \omega_{l}\left(\varepsilon_{\infty}^{-1}-\varepsilon_{s}^{-1}\right)}{4 a h c B \sqrt{\varepsilon^{\prime}}}\left(\frac{E_{A, 1}}{E_{A, 1}-\omega}\right)^{2} \\
\times\left[\frac{M_{||} B}{\mu_{\perp} \Delta}\right]^{1 / 2} N\left(\omega_{l}\right) I(\omega), \\
\alpha_{2}(\omega)=\frac{e^{2} \varepsilon_{s} \beta_{A, 1} \omega_{l}^{2}\left(\varepsilon_{\infty}^{-1}-\varepsilon_{s}^{-1}\right)^{2}}{16 a h c B^{2} \sqrt{\varepsilon^{\prime}}}\left(\frac{E_{A, 1}}{E_{A, 1}-\omega}\right)^{2} \\
\times\left[\frac{M}{\mu}\right]^{3}\left(\frac{\left\langle E_{A, n^{\prime}}\right\rangle-E_{A, 1}}{\left\langle E_{A, n^{\prime}}\right\rangle-\omega}\right)^{2} N^{2}\left(\omega_{l}\right) \sum_{n} I_{n}(\omega),
\end{gathered}
$$

respectively. The definitions and physical meanings of various quantities can be referred to in Refs. 15, 16, and 18 . Under certain conditions, the transition probabilities for emissions are related to the absorption coefficients by ${ }^{10}$ 


$$
W_{e m}(\omega) \propto e^{-\omega / k_{B} T} \alpha_{n}(\omega), \quad n=1,2 .
$$

In the present work, we are mainly concerned with the influence of the effective mass of free excitons, in particular, heavy hole on the first- and second-order PSBs in $\mathrm{ZnO}$. In Eqs. (1) and (2), $M_{\|}$is the total effective mass of free excitons in the $c$ axis of $\mathrm{ZnO}, \mu_{\perp}$ the reduced effective mass in the direction perpendicular to the $c$ axis, $M=m_{e}+m_{h}$ the total effective mass of free exciton, and $\mu=m_{e} m_{h} /\left(m_{e}=m_{h}\right)$ the reduced effective mass of free exciton. Considering the anisotropic properties of $\mathrm{ZnO}$ with wurtzite structure, $m_{e(h)}=\left[m_{e(h) \|} \times m_{e(h) \perp}^{2}\right]^{1 / 3}$ is taken in the calculations. ${ }^{15,16,18}$ In addition, $I(\omega)$ and $I_{n}(\omega)$ in Eqs. (1) and (2), respectively, are also related to the effective mass of free exciton. ${ }^{15,16,18}$ Therefore, the effective mass of free exciton and thus heave hole has important influence on the first- and second-order PSBs of free exciton in $\mathrm{ZnO}$.

\section{RESULTS AND DISCUSSION}

Figure 1 shows that the theoretical absorption spectra (lines plus solid circles) and the emission curves (solid lines) of the first- and second-order LO phonon-assisted transitions of free excitons in $\mathrm{ZnO}$ at $146 \mathrm{~K}$. The parameters used in the calculations are listed in Table I. From the figure, it can be seen that the absorption spectra for the optical transitions involving one or two LO phonons have two distinct onset points at energies of $E_{A, 1}-\omega_{l}$ or $E_{A, 1}-2 \omega_{l}$, respectively. Here $E_{A, 1}$ and $\omega_{l}$ represent the energy of the ZPL of free excitons and the characteristic energy of LO phonons at the zone center in $\mathrm{ZnO}$. However, the distinct peak positions of 1LO- and 2LO-PSBs obviously departure from $E_{A, 1}-\omega_{l}$ and $E_{A, 1}-2 \omega_{l}$, respectively. In other words, the energy spacings from the peaks of 1LO- and 2LO-PSBs to the ZPL deviate from one and two units of LO phonon characteristic energy, respectively. For example, the energy spacings for 1LO- and 2LOPSBs are 58.2 and $135.3 \mathrm{meV}$ with respect to $E_{A, 1}=3.3647$ $\mathrm{eV}$ at $146 \mathrm{~K}$, respectively. In particular, the energy spacing for 1LO-PSB is significantly smaller than the standard

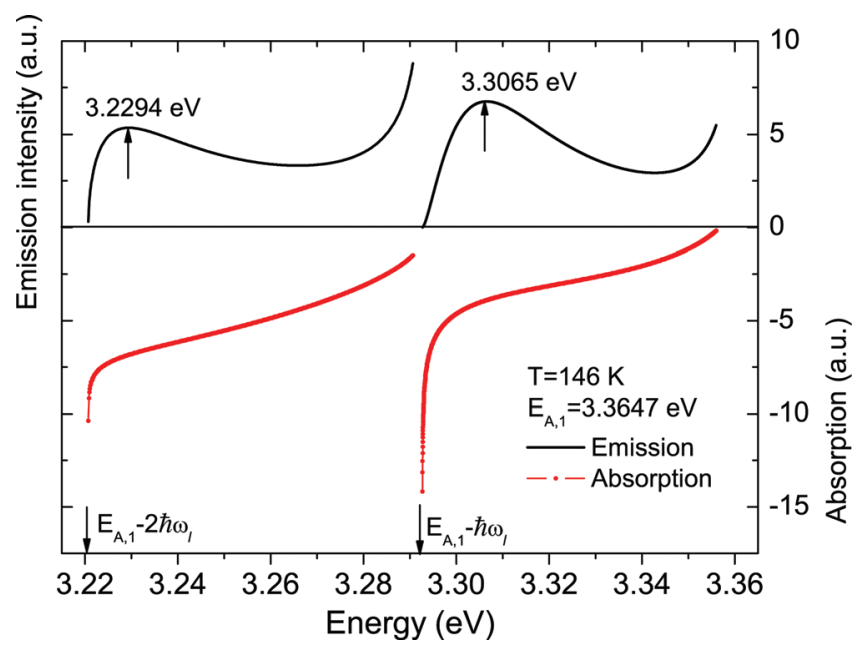

FIG. 1. (Color online) The solid lines are the calculated emission spectra of free excitons involving one or two LO phonons in $\mathrm{ZnO}$ at $146 \mathrm{~K}$ while the lines plus symbols represent the calculated absorption spectra (logarithmic scale) at the same temperature.
TABLE I. Parameters used in the calculations of the absorption coefficients.

\begin{tabular}{lll}
\hline \hline Parameters & Symbol & ZnO \\
\hline Electron effective mass (unit of $\left.m_{0}\right)$ & $m_{e}$ & $0.24^{\mathrm{a}}$ \\
Hole effective mass (unit of $m_{0}$ ) & $m_{h \perp}$ & 0.8 \\
& $m_{h \|}$ & 5 \\
Exciton binding energy (meV) & $B$ & $60^{\mathrm{b}}$ \\
LO phonon energy (meV) & $\eta \omega_{1}$ & $72^{\mathrm{a}}$ \\
Statistic dielectric constant & $\varepsilon s=\left(\varepsilon_{\perp} \varepsilon_{\|}\right)^{1 / 2}$ & $7.88^{\mathrm{a}}$ \\
High-frequency dielectric constant & $\varepsilon_{\infty}=n_{\perp} n_{\|}$ & $4.0^{\mathrm{a}}$ \\
Dielectric constant at $E_{A, 1}$ in the & $\varepsilon^{\prime}$ & $6.2^{\mathrm{a}}$ \\
$\quad$ & & \\
absence of $n=1$ exciton & $4 \pi \beta_{A, 1}$ & $0.0066^{\mathrm{a}}$ \\
$n=1 A, B$, or $C$ exciton $(\varepsilon \perp c)$ & & \\
& $4 \pi \beta_{B, 1}$ & $0.0227^{\mathrm{a}}$ \\
& $4 \pi \beta_{C, 1}$ & $0.0300^{\mathrm{a}}$ \\
\hline \hline
\end{tabular}

${ }^{\mathrm{a}}$ Reference 15 .

${ }^{\mathrm{b}}$ Reference 6 .

energy unit of LO phonons. Furthermore, both PSBs exhibit unique asymmetric line shape. In fact, these spectral features of PSBs basically reflect the thermal distribution of free excitons at their higher excited states that make important contributions to the phonon-assisted radiative recombination of free excitons at higher temperatures. ${ }^{16-18}$

Now we turn to discuss the experimental results and direct comparison with the theoretical curves. In Fig. 2, we compare the theoretical emission curves (solid lines) and measured luminescence spectra (thin solid lines plus solid circles) at three typical different temperatures of 116, 136, and $146 \mathrm{~K}$. The ZPL lines of free excitons and bound excitons are donated by FXA and $\mathrm{D}^{0} \mathrm{X}$, respectively. The 1LOand $2 \mathrm{LO}-\mathrm{PSB}$ of free excitons are marked by $1 \mathrm{LO}$ and $2 \mathrm{LO}$ in the figure, respectively. The phonon sidebands of bound excitons have become very weak at the moderately high temperature range. Considering the complexity of many-body (photon-exciton-phonon) interactions in the case studied in the present work, agreement between theory and experiment is reasonably good. It should be noted that only the heavyhole effective mass is adjustable parameter in the theoretical calculations. Our experimental luminescence spectra at higher temperatures (not shown here) also support Shan et al.'s conclusion that 1LO-PSB gradually becomes dominant over its parents line- the ZPL line with increasing temperature. It is difficult to find the "peaks" of PSBs at temperatures higher than $180 \mathrm{~K}$ due to the strong asymmetric broadening of the PSBs, especially 1LO-PSB that is strongly overlapping with the ZPL line at these higher temperatures. At low temperature range lower than $116 \mathrm{~K}$, although the peak positions of PSBs between theory and experiment agree quite well, the theoretical line shape of PSBs, especially 2LO-PSB, is wider than the experimental spectra. A possible major factor causing such discrepancy is the Boltzmann distribution approximation of free excitons adopted in the present calculations. In fact, the exciton gas obeys Bose-Einstein statistics of quantum mechanics. Therefore, adopting Boltzmann statistics for the exciton gas in the calculation of the emission spectra from the absorption coefficients [i.e., Eq. 


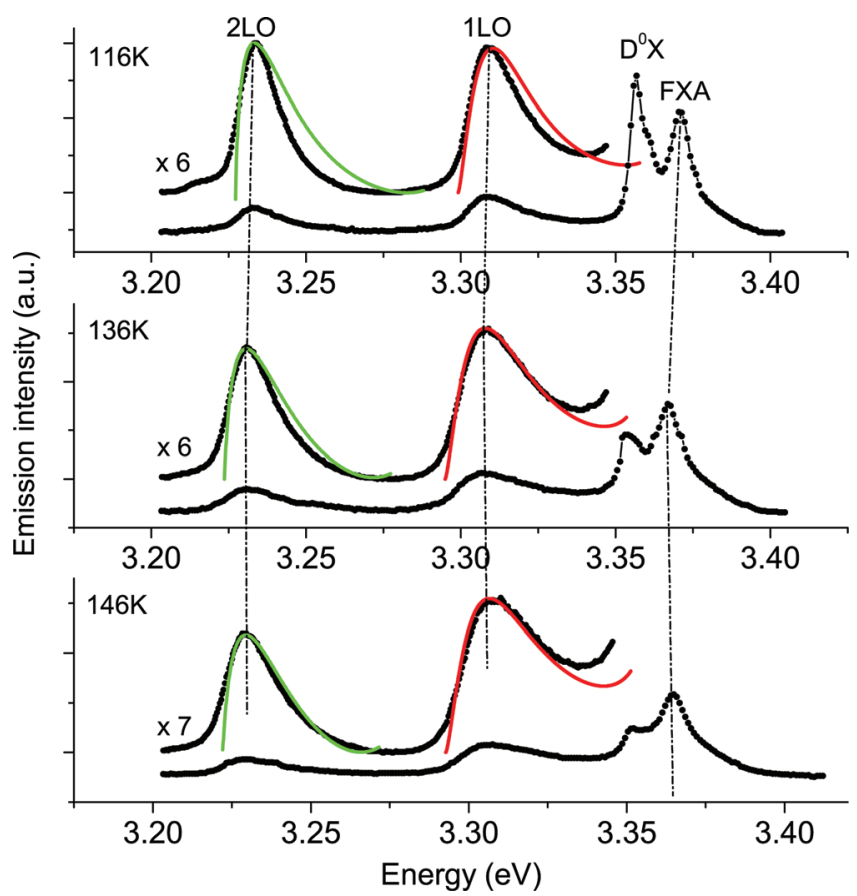

FIG. 2. (Color online) Measured PL spectra (lines plus solid circles) of $\mathrm{ZnO}$ at three different temperatures of 116,136 , and $146 \mathrm{~K}$. The phonon sideband portions of the spectra were enlarged for a closer look and better comparison to the calculated emission spectra (solid lines). The ZPL lines of free excitons and bound excitons are donated by FXA and $\mathrm{D}^{0} \mathrm{X}$, respectively, whereas the 1LO- and 2LO-PSB of free excitons are marked by $1 \mathrm{LO}$ and 2LO in the figure, respectively. The dashed-dotted lines are used to guide the eyes to look at the peak position variation of various emissions at different temperatures.

(3)] could lead to overestimation of the line shape of emission spectra, in particular, at low temperatures. For the calculation of 2LO-PSB, more approximations have to be introduced due to increase of complexity and difficulty in the mathematic treatment. ${ }^{15,16}$ These approximations could be responsible for the a bit more deviation of theoretical 2LOPSB spectra from the experimental spectra at low temperatures. In addition, the influence of the bound exciton related emissions should be mentioned. At low temperatures below around $80 \mathrm{~K}$, the LO phonon sidebands of bound excitons are relatively stronger. ${ }^{19}$ Although they located at the lower energy side of the corresponding phonon sidebands of free excitons and are well resolved, the appearance of these peaks still at a certain content hinders the accurate theoretical interpretation of the phonon sidebands of free excitons under study. Fortunately, the intensities of the phonon sidebands of bound excitons rapidly drop with increasing temperature, so that at moderately high temperatures, the LO phonon sidebands of free excitons can be well quantitatively calculated using the S-M theory. It is worth mentioning another interesting phenomenon that 2LO-PSB is unusually strong. For example, it is even stronger than 1LO-PSB at temperatures below $116 \mathrm{~K}$. In GaN, such phenomenon is at least not substantial. ${ }^{20}$ The physical origin causing the unusual phenomenon has not yet understood and is being investigated.

The calculated (lines plus various solid symbols) and measured (lines plus open circles) energy spacings of 1LOand 2LO-PSB with respect to the ZPL line versus temperature are depicted in Fig. 3, respectively. The theoretical results agree quite well with the experimental data. It should be noted that the energy spacing for 2LO-PSB shown in the figure has been divided by two so that it can be directly compared with the standard LO phonon energy $(\omega 1=72 \mathrm{meV}$ marked by a horizontal arrow) and the energy spacing for 1LO-PSB. From Fig. 3, it can be seen that as the temperature increases, the energy spacings of both PSBs gradually deviate from the standard LO phonon energy. Moreover, the deviation rate of 1LO-PSB is much higher than that of 2LO-PSB. Similar phenomenon has been observed in wurtzite GaN. ${ }^{20}$ For this phenomenon, it can be clearly explained from an approximate expression for the free-exciton emission probability involving $m$ LO phonons. In the energy range near the threshold (i.e., $E_{A, 1}-m \omega_{l}$ ), the emission probability can be approximated as $W_{e m}(\omega) \propto \Delta^{(5 / 2)-m} \mathrm{e}^{-\Delta / \mathrm{ksT}}$ with $\Delta=\omega$ $-\left(E_{A, 1}-m \omega_{1}\right){ }^{16}$ From this approximated expression, it is not difficult to prove that the temperature dependence of the peak positions of 1LO- and 2LO-PSB is $E_{A, 1}-\omega_{1}+(3 / 2) k_{B} T$ and $E_{A, 1}-2 \omega_{1}+(1 / 2) k_{B} T$, respectively. ${ }^{20,21}$ So, the energy spacings of 1LO- and 2LO-PSB departure from the LO phonon energy at rates of $-(3 / 2) k_{B} T$ and $-(1 / 4) k_{B} T$, respectively, as the temperature increases. ${ }^{20,22}$

As mentioned earlier, the effective mass of heavy holes is the only one adjustable parameter in the calculations. Actually, the effective mass of heavy holes in $\mathrm{ZnO}$ is anisotropic due to the hexagonal symmetry of crystal structure. We also theoretically examine influence of the heavy-hole effective mass on the energy spacings of 1LO- and 2LO-PSB with respect to the ZPL line. In general, the temperature dependence of both PSBs becomes weaker with increasing the heavy-hole effective mass. As listed in Table I, when $m_{h \perp}=0.8 m_{0}$ and $m_{h \|}=5.0 m_{0}$ are adopted, the excellent agreement between theory and experiment is achieved in the interested temperature range from 6 to $150 \mathrm{~K}$. It indicates that the $m_{h \|}$ of $\mathrm{ZnO}$ should be much larger than the rest mass

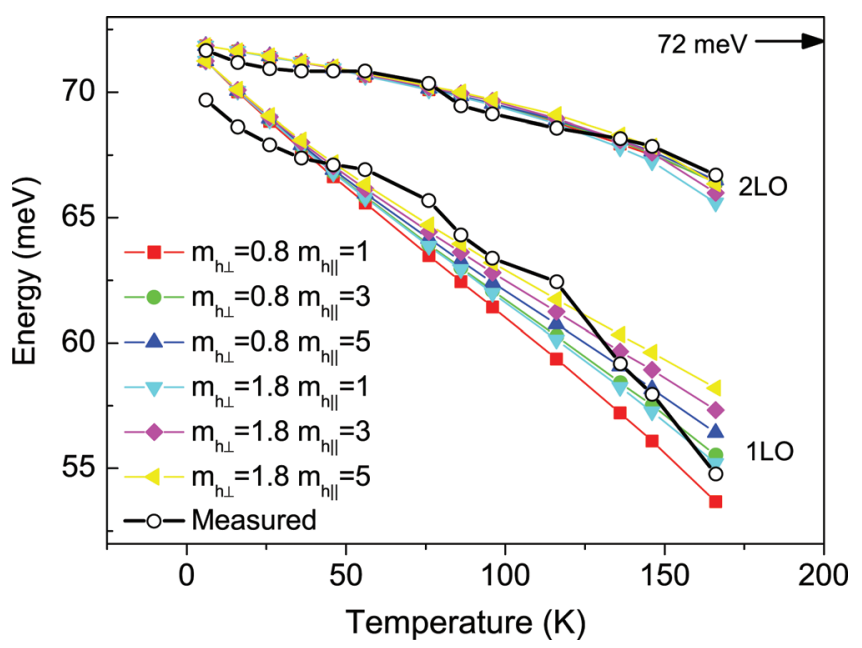

FIG. 3. (Color online) Measured (lines plus open circles) and calculated (lines plus various solid symbols) energy spacings of 1LO- and 2LO-PSB with respect to the ZPL line, respectively, as a function of temperature. Note that the energy spacing for 2LO-PSB shown in the figure is half the actual energy spacing of 2LO-PSB relative to the ZPL for direct comparison to the standard LO phonon energy. 
of free electron $m_{0}$, which is in agreement with the latest theoretical results. $^{23,24}$

\section{CONCLUSION}

In conclusion, a combined theoretical and experimental investigation on the temperature dependence of LO phonon sidebands of free excitons in $\mathrm{ZnO}$ is presented. The SegallMahan theory taking exciton-LO phonon and exciton-photon coupling into account is employed here to quantitatively interpret experimental spectra in a wide temperature range. The effective mass of heavy holes in $\mathrm{ZnO}$ is then determined. Despite this, the new finding still calls for further investigations on the exciton-phonon interactions in $\mathrm{ZnO}$.

\section{ACKNOWLEDGMENTS}

The authors gratefully acknowledge G. Q. Li for his contribution to the calculation program coding and also wish to thank W. K. Ho for providing $\mathrm{ZnO}$ samples used in the present study. The work was financially supported by RGCGRF Grant of HKSAR (Grant No. HKU 7056/06P) and the Joint Research Fund for Overseas Chinese, Hong Kong and Macau Scientists of NSFC (Grant No. 61028012).

${ }^{1}$ M. H. Huang, S. Mao, H. Feick, H. Q. Yan, Y. Y. Wu, H. Kind, E. Weber, R. Russo, and P. Yang, Science 292, 1897 (2001).

${ }^{2}$ D. C. Reynolds, D. C. Look, and B. Jogai, Solid State Commun. 99, 873 (1996).
${ }^{3}$ D. M. Bagnall, Y. F. Chen, Z. Zhu, T. Yao, S. Koyama, M. Y. Shen, and T. Goto, Appl. Phys. Lett. 70, 2230 (1997).

${ }^{4}$ H. Cao, Y. G. Zhao, H. C. Ong, S. T. Ho, J. Y. Dai, J. Y. Wu, and R. P. H. Chang, Appl. Phys. Lett. 73, 3656 (1998).

${ }^{5}$ D. C. Reynolds, D. C. Look, B. Jogai, C. W. Litton, G. Cantwell, and W. C. Harsch, Phys. Rev. B 60, 2340 (1999).

${ }^{6}$ W. Y. Liang and A. D. Yoffe, Phys. Rev. Lett. 20, 59 (1967).

${ }^{7}$ D. C. Look, Mat. Sci. Eng. B 80, 383 (2001).

${ }^{8}$ D. G. Thomas, J. Phys. Chem. Solids 15, 86 (1960).

${ }^{9}$ J. J. Hopfield, J. Phys. Chem. Solids 15, 97 (1960).

${ }^{10}$ R. E. Dietz, J. J. Hopfield, and D. G. Thomas, J. Appl. Phys. 32, 2282 (1961).

${ }^{11}$ Y. S. Park, C. W. Litton, T. C. Collins, and D. C. Reynolds, Phys. Rev. 143, 512 (1966).

${ }^{12}$ W. Shan, W. Walukiewicz, J. W. Ager III, K. M. Yu, H. B. Yuan, H. P. Xin, G. Cantwell, and J. J. Song, Appl. Phys. Lett. 86, 191911 (2005).

${ }^{13}$ S. Bethke, H. Pan, and B. W. Wessels, Appl. Phys. Lett. 52, 138 (1988).

${ }^{14}$ D. C. Reynolds, D. C. Look, B. Jogai, J. E. Hoelscher, R. E. Sherriff, M. T. Harris, and M. J. Callahan, J. Appl. Phys. 88, 2152 (2000).

${ }^{15}$ B. Segall, Phys. Rev. 163, 769 (1967); ibid. 150, 734 (1966).

${ }^{16}$ B. Segall and G. D. Mahan, Phys. Rev. 171, 935 (1968).

${ }^{17}$ S. J. Xu, G. Q. Li, S.-J. Xiong, S. Y. Tong, C. M. Che, W. Liu, and M. F. Li, J. Chem. Phys. 122, 244712 (2005).

${ }^{18}$ S. J. Xu, G. Q. Li, S.-J. Xiong, and C. M. Che, J. Appl. Phys. 99, 073508 (2006).

${ }^{19}$ S. J. Xu, S.-J. Xiong, and S. L. Shi, J. Chem. Phys. 123, 221105 (2005).

${ }^{20}$ S. J. Xu, W. Liu, and M. F. Li, Appl. Phys. Lett. 77, 3376 (2000).

${ }^{21}$ C. Klingshirn, Phys. Status Solidi B 71, 547 (1975).

${ }^{22}$ S. J. Xu, W. Liu, and M. F. Li, Appl. Phys. Lett. 81, 2959 (2002).

${ }^{23}$ W. J. Fan, J. B. Xia, P. A. Agus, S. T. Tan, S. F. Yu, and X. W. Sun, J. Appl. Phys. 99, 013702 (2006).

${ }^{24}$ S. L. Shi, G. Q. Li, S. J. Xu, Y. Zhao, and G. H. Chen, J. Phys. Chem. B 110, 10475 (2006). 